\title{
Gender Differences in Predictors of Heart Failure Morbidity and Mortal- ity in an Urban Swedish Population: The Malmö Preventive Project
}

\author{
G. Tasevska-Dinevska*, ${ }^{* 1}$ L.M.A. Kennedy ${ }^{2}$, D. Anevski ${ }^{3}$, P. Nilsson ${ }^{2}$, A. Christensson ${ }^{4}$ and \\ R. Willenheimer ${ }^{2,5}$
}

${ }^{1}$ Lund University, Department of Cardiology; ${ }^{2}$ Department of Clinical Sciences Medicine; ${ }^{3}$ Malmö, and Lund Univer-
sity, Centre for Mathematical Sciences; ${ }^{4}$ Dept of Nephrology and Transplantation; ${ }^{5}$ University Hospital Malmö, Heart
Health Group, Sweden

Abstract: Objective/Background: Reports on heart failure (HF) predictors are scarce. We assessed gender-specific HF predictors.

Design: Preventive case-finding programme, register study.

Setting: City population-based sample.

Methods: We examined 33,342 HF-free subjects, 32.7\% women, included in Malmö Preventive Project. Mean inclusion age was $49.7 \pm 7.4$ years for women and $43.7 \pm 6.6$ years for men.

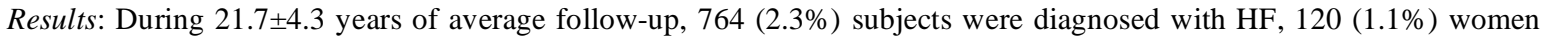
and $644(2.9 \%)$ men. Following bootstrap analysis, the only strong independent predictor of HF among women was smoking. Independent predictors of $\mathrm{HF}$ among men were diastolic blood pressure (BP), fasting blood-glucose, smoking, family history of myocardial infarction, and previous cardiovascular disease (CVD). During follow-up, 5,370 (16.1\%) subjects died, $978(9.0 \%)$ women and 4,392 (19.6\%) men. Among both women and men, strong independent predictors of combined HF or all-cause death were high serum-triglycerides, fasting blood-glucose and estimated glomerular filtration rate, smoking, and previous CVD. Among men, also underweight, high BMI, and systolic and diastolic BP, were strong independent predictors of HF or death.

Conclusions: Although women and men shared many predictors of HF, there were several important differences between sexes.

Keywords: Heart failure, mortality, predictors, men, women.

\section{INTRODUCTION}

The burden of cardiovascular disease (CVD), including heart failure (HF), is huge among both women and men in the Western world and CVD is the leading cause for morbidity and mortality [1-5]. The most important risk factors for CVD are hypertension, dyslipidaemia, smoking, diabetes, psychosocial stress, lack of physical exercise, abdominal obesity and high levels of inflammatory markers [6-9]. Wilhelmsen et al. [10] reported long-term data showing a remaining high CVD risk related to self-reported diabetes and family history of CVD among first degree relatives. Similar results have been reported from the Reykjavik Cohort Study [11]. The INTERHEART study showed that, of all first myocardial infarctions (MI), 90\% in men and $94 \%$ in women could be explained by nine risk factors; dyslipidemia, hypertension, diabetes, smoking, abdominal obesity, poor psychosocial environment, lack of regular alcohol intake, physical inactivity and inadequate intake of fresh fruit and vegetables $[12,13]$. The Copenhagen City Heart Study [14] showed that smoking, hypertension and lack of daily alcohol intake

*Address correspondence to this author at the Department of Cardiology, University Hospital Malmö, S-20502 Malmö, Sweden; Tel: +46 40337194; Fax: +46 40336209; E-mail: Gordana.Tasevska@med.lu.se conferred the highest population-attributable risk for coronary heart disease among men, whereas smoking, hypertension and hypercholesterolaemia were most important among women. Nilsson et al. showed that the strongest risk factor for coronary events among men was smoking, followed by hypercholesterolaemia and diabetes, whereas smoking, diabetes and hypertension were most important for women [15].

If the known predictors of CVD also are predictors of HF is not well known, since few studies have assessed predictors of HF [16-21]. The present analysis was performed in a large population sample, the Malmö Preventive project [22], with the primary aim of identifying gender-specific baseline predictors of HF.

\section{METHODS}

\section{Study Design}

The Malmö Preventive Project (MPP) was launched in 1974 at the University Hospital in Malmö, the third largest city in Sweden [22]. The project was a preventive casefinding programme with the aim to screen for CVD risk factors, alcohol abuse and breast cancer in the population. Birth cohorts of inhabitants in Malmö, born 1921-1949, were invited to participate. Men were mostly screened in the first half of the period (1974-1982) and women in the latter half 
(1981-1992), resulting in shorter mean follow-up time for women. A total of 12,142 women and 30,818 men were invited and 33,346 middle-aged individuals (33\% women) attended the screening programme. Thus, the overall attendance rate was $71 \%$ (range $64 \%-78 \%$ ) [22]. At the time of inclusion, subjects underwent blood sampling (after an overnight fast), supine BP measurement and anthropometric measurements, and filled out questionnaires. Patients who were hospitalised for, or died of HF during follow-up, were considered HF patients. The following ICD codes in the first position were used to classify subjects as HF patients: ICD9, 428; ICD10, I500, I501 and I509. At baseline, four patients had a diagnosis of HF and these were excluded from all analyses. A diagnosis of HF (first position) was based on a clinical assessment, X-ray examination and, to various extents, echocardiography, according to the clinical standards of the different time periods during follow-up. In some cases the diagnosis was based on autopsy. Death and hospitalisation diagnoses were obtained from the Swedish national registries. An internal HF diagnosis validation was performed in a random sample of $42(5.5 \%)$ of the HF patients and in 300 (0.9\%) subjects without a HF diagnosis, included in the Malmö Prevention Project. Patients were categorised according to body mass index (BMI); underweight <22.00, normalweight 22.00-24.99, overweight 25.00-29.99, and obesity $\geq 30.00 \mathrm{~kg} / \mathrm{m}^{2}$ [23]. Hypertension was defined as BP $\geq 140 / 90$ $\mathrm{mmHg}$. Estimated glomerular filtration rate (eGFR) was calculated according to the Modification of Diet in Renal Disease (MDRD) study formula [24].

\section{Subjects}

Between 1974 and 1992, a total of 33,342 subjects who did not have a history of HF were included in the present study; 10,900 women and 22,442 men. Mean age at inclusion was $45.7 \pm 7.4$ years for all subjects; $49.7 \pm 7.4$ years for women and $43.7 \pm 6.6$ years for men. The $31^{\text {st }}$ of December 2002 was the study end and subjects being alive were censored on this date. Subjects were censored on the day of the last available reliable data on morbidity and mortality if that occurred before the $31^{\text {st }}$ of December 2002, e.g. if pa-

Table 1. Baseline Data

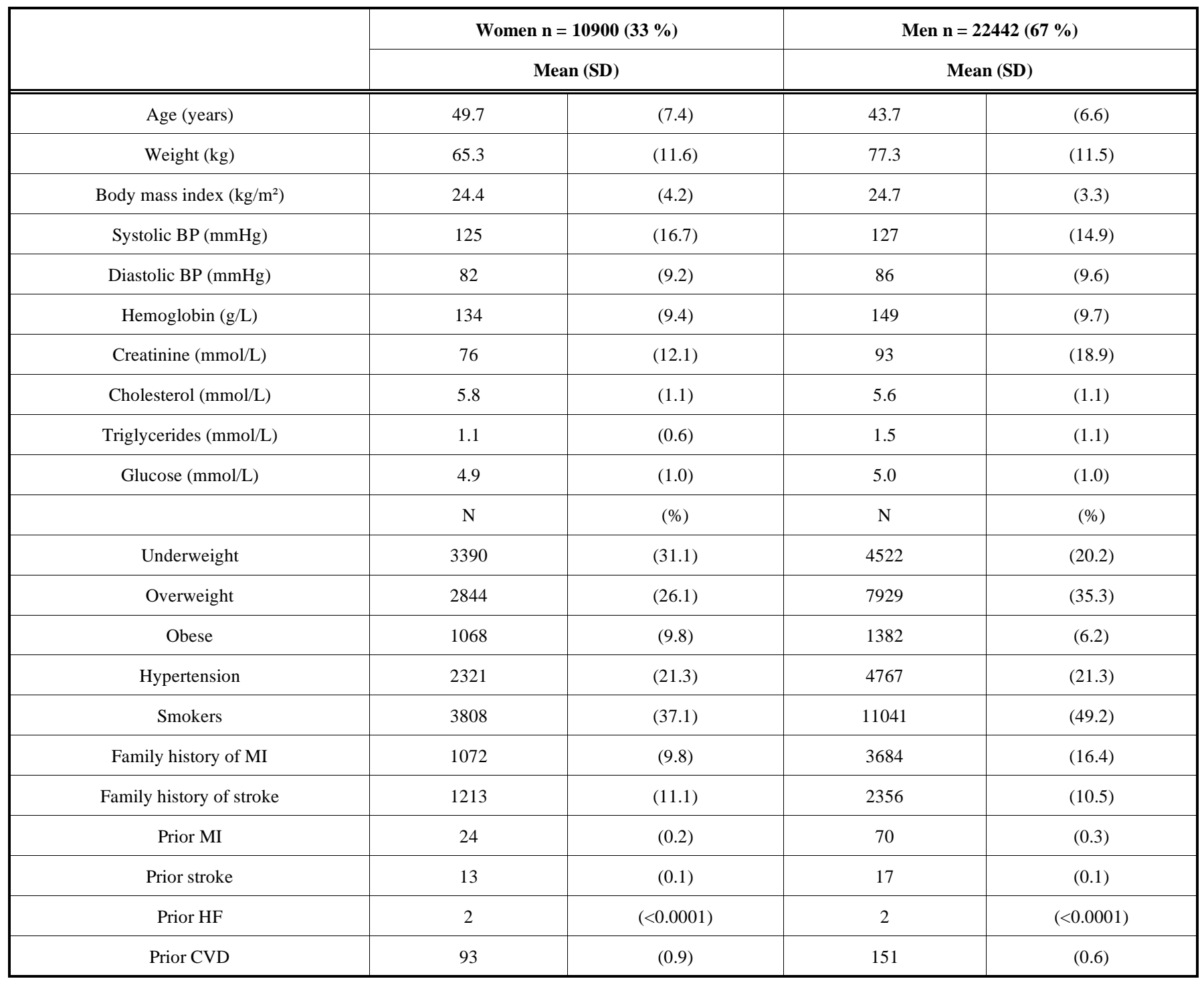

BP, Blood pressure; MI, Myocardial infarction; HF, Heart failure; CVD, Cardiovascular disease. 
tients moved to another country. In such patients, the censor date constituted the study end. Subjects were included on average $21.7 \pm 4.3$ years before study end; $18.2 \pm 4.3$ years for women and $23.3 \pm 3.0$ years for men.

\section{Statistics}

Separately in women and men, all baseline variables were first analysed in univariable Cox regression models, with a diagnosis of $\mathrm{HF}$ and combined HF or all-cause death as dependent endpoint variables, respectively. The basic time variable used was age at the time of the endpoint, with data treated as left-truncated to adjust for time. The tested baseline variables were BMI, BMI group (underweight, overweight and obesity, respectively, versus normal weight), systolic and diastolic BP, hypertension, blood (b)haemoglobin, eGFR, serum (s)-cholesterol, s-triglycerides, fasting (f) b-glucose, sex, smoking status (current/previous smoking versus never smoking), age, family history of MI, and previous CVD. The results were expressed as hazard ratio (HR) and 95\% confidence interval (CI). HRs for continuous variables are given per unit increase, except for BMI, $\mathrm{BP}$ and eGFR, where HRs are per 10 units increase. $\mathrm{P}<0.05$ denotes statistical significance. All variables were further tested for inclusion in a multivariable analysis. The multivariable Cox regression models were chosen using backward stepwise deletion of variables. The final models were tested using likelihood ratio tests. Bootstrap analyses [25] were performed to validate the final model, using the more robust technique of resampling of the full information of the individuals, see [26] for other resampling techniques. The bootstrap analyses were performed by 100 resamplings from the original sample, and for each resampled data set a stepwise backward deletion of variables analysis was performed in a multivariable Cox regression model. The results of the bootstrap analyses are presented as frequency of inclusion of covariates in a final multivariate regression model. Covariates with frequencies $>90 \%$ were labelled as strong independent predictors and covariates with frequencies $>30 \%$ but $<90 \%$ were considered weak independent predictors [27]. Final multivariable Cox regression models including only strong and both weak and strong independent predictors, respectively, are presented. The proportional hazards assumptions were tested using residuals based test [28]. All survival analyses were performed using the package Survival in R, www.r-project.org.

\section{RESULTS}

\section{Baseline Characteristics, Morbidity and Mortality}

Baseline characteristics are shown in Table 1. For the two endpoints, median time to endpoint was 8016 days (21.9 years) for HF and 6142 days (16.8 years) for combined HF or death. Interquartile range (IQR) was 2257 (6.2 years) for

Table 2. Baseline Predictors of Heart Failure and Combined Heart Failure or Death in Women and Men (Adjusted for Age). Univariable Analysis

\begin{tabular}{|c|c|c|c|c|c|c|c|c|c|c|c|c|}
\hline & \multicolumn{6}{|c|}{ Predictors of HF } & \multicolumn{6}{|c|}{ Predictors of HF or Death } \\
\hline & HR & $95 \% \mathrm{CI}$ & $\mathbf{P}$ & HR & $95 \% \mathrm{CI}$ & $\mathbf{P}$ & HR & $95 \% \mathrm{CI}$ & $\mathbf{P}$ & HR & $95 \% \mathrm{CI}$ & $\mathbf{P}$ \\
\hline $\begin{array}{c}\text { BMI } \\
\mathrm{kg} / \mathrm{m}^{2}\end{array}$ & 1.11 & $1.07-1.15$ & $<.0001$ & 1.13 & $1.10-1.15$ & $<.0001$ & 1.02 & $1.01-1.04$ & $<.0001$ & 1.04 & $1.03-1.05$ & $<.0001$ \\
\hline Obesity & 3.68 & $2.43-5.57$ & $<.0001$ & 2.82 & $2.27-3.50$ & $<.0001$ & 1.41 & $1.18-1.69$ & $<.0001$ & 1.67 & $1.51-1.84$ & $<.0001$ \\
\hline Systolic BP mm Hg & 1.02 & $1.01-1.03$ & $<.0001$ & 1.02 & $1.02-1.03$ & $<.0001$ & 1.01 & $1.01-1.01$ & $<.0001$ & 1.01 & $1.01-1.01$ & $<.0001$ \\
\hline Hypertension & 1.91 & $1.29-2.82$ & $<.0001$ & 2.17 & $1.84-2.55$ & $<.0001$ & 1.37 & $1.19-1.57$ & $<.0001$ & 1.50 & $1.41-1.60$ & $<.0001$ \\
\hline Cholesterol mmol/L & 1.13 & $0.95-1.35$ & 0.18 & 1.21 & $1.12-1.30$ & $<.0001$ & 1.10 & $1.04-1.16$ & $<.0001$ & 1.11 & $1.09-1.13$ & $<.0001$ \\
\hline Triglycerides mmol/L & 1.84 & $1.52-2.22$ & $<.0001$ & 1.26 & $1.20-1.33$ & $<.0001$ & 1.44 & $1.33-1.56$ & $<.0001$ & 1.12 & $1.10-1.15$ & $<.0001$ \\
\hline Glucose mmol/L & 1.21 & $1.10-1.33$ & $<.0001$ & 1.20 & $1.15-1.25$ & $<.0001$ & 1.19 & $1.15-1.24$ & $<.0001$ & 1.13 & $1.11-1.15$ & $<.0001$ \\
\hline Sex: Women & - & - & - & - & - & - & - & - & - & - & - & - \\
\hline Smoking & 2.46 & $1.68-3.59$ & $<.0001$ & 1.68 & $1.43-1.98$ & $<.0001$ & 2.13 & $1.87-2.42$ & $<.0001$ & 2.04 & $1.97-2.17$ & $<.0001$ \\
\hline FH of MI & 2.24 & $1.36-3.69$ & $<.0001$ & 1.45 & $1.19-1.75$ & $<.0001$ & 1.16 & $0.95-1.42$ & 0.16 & 1.11 & $1.03-1.20$ & 0.01 \\
\hline Prior CVD & 6.00 & $2.43-14.83$ & $<.0001$ & 4.78 & $2.92-7.83$ & $<.0001$ & 2.93 & $1.92-4.47$ & $<.0001$ & 2.59 & $2.07-3.25$ & $<.0001$ \\
\hline
\end{tabular}

HF, Heart failure; BMI, Body mass index; BP, Blood pressure; Smoking, current and previous smokers versus never smokers; FH of MI, Family history of acute myocardial infarction; CVD, Cardiovascular disease, eGFR (Estimated glomerular filtration rate).

HRs for continuous variables are per unit increase, except for BMI, BP and eGFR, where HRs are per 10 units increase. HRs for the baseline BMI categories are for the respective categories versus the normal weight category. HRs for all other categories are compared to the opposite category. 
HF and 2672 days (7.3 years) for HF or death. During an average follow-up of $21.7 \pm 4.3$ years from inclusion, 764 (2.3\%) subjects were diagnosed with $\mathrm{HF}, 120$ (1.1\%) women and $644(2.9 \%)$ men. In all, 5,370 (16.1\%) subjects died, 978 $(9.0 \%)$ women and 4,392 (19.6\%) men.

\section{Internal Validation of the HF Diagnosis and the Lack of a HF Diagnosis}

An internal validation of the HF diagnosis was done in $42(5.5 \%)$ HF patients, 5 women and 37 men, randomly selected over the entire follow-up period. Among these, 39 (93\%) had data on left ventricular ejection fraction (LVEF) and $35(83 \%)$ of these had a record of symptoms of HF. Mean LVEF was 34\% (range 10-60\%), and LVEF was $>50 \%$ in 5 and $>40 \%$ in 10 patients. Those who had not undergone an echocardiogram $(n=3)$, all had typical HF symptoms and typical HF oedema on pulmonary X-ray examination, and all of those who had normal LVEF had typical HF symptoms and echocardiographic signs of LV diastolic dysfunction. An internal validation of the non-HF diagnosis was done in $300(0.9 \%)$ subjects, 90 women and 210 men, randomly selected over the entire follow-up period. Among these, 269 (90\%) subjects, 89 women and 180 men, had no hospitalisation or HF diagnosis during follow-up. There was no indication of $\mathrm{HF}$ in the 31 patients with a hospitalisation during follow-up, although 7 (3 women) had a HF diagnosis in the $3^{\text {rd }}$ or $4^{\text {th }}$ position at one occasion, due to transitory symptoms indicating temporary HF in connection with one single hospitalisation. The HF diagnosis was not mentioned again during follow-up after that hospitalisation.

\section{Predictors of Developing HF}

Most tested baseline variables were predictors of development of $\mathrm{HF}$ and combined HF or death in univariable analysis (Table 2).

Among women, bootstrap analysis showed that only smoking was a strong independent predictor of developing HF (Table 3). Obesity, high BMI, s-triglycerides and fbglucose, as well as family history of MI, and previous CVD, were weak independent predictors of HF (Table 3). In the final multivariable model including both strong and weak independent predictors, high s-triglycerides, family history of MI, and previous CVD, were independent predictors (Table $4 \mathbf{b})$.

Among men, high diastolic BP and fb-glucose, as well as smoking, family history of MI, and previous CVD were strong independent predictors of developing HF (Table 3 ). The same variables were independent predictors of HF in the final multivariable model including only strong independent predictors (Table 4a). In the final multivariable model including both strong and weak independent predictors, also high BMI, systolic BP and s-cholesterol, were independent predictors of HF among men (Table $\mathbf{4 b}$ ).

Table 3. Baseline Predictors of Heart Failure and Combined Heart Failure or Death in Men and Women According to Bootstrap Analysis. Percent of Random Sub-Sample Multivariable Analyses where the Respective Variables were Independent are Given

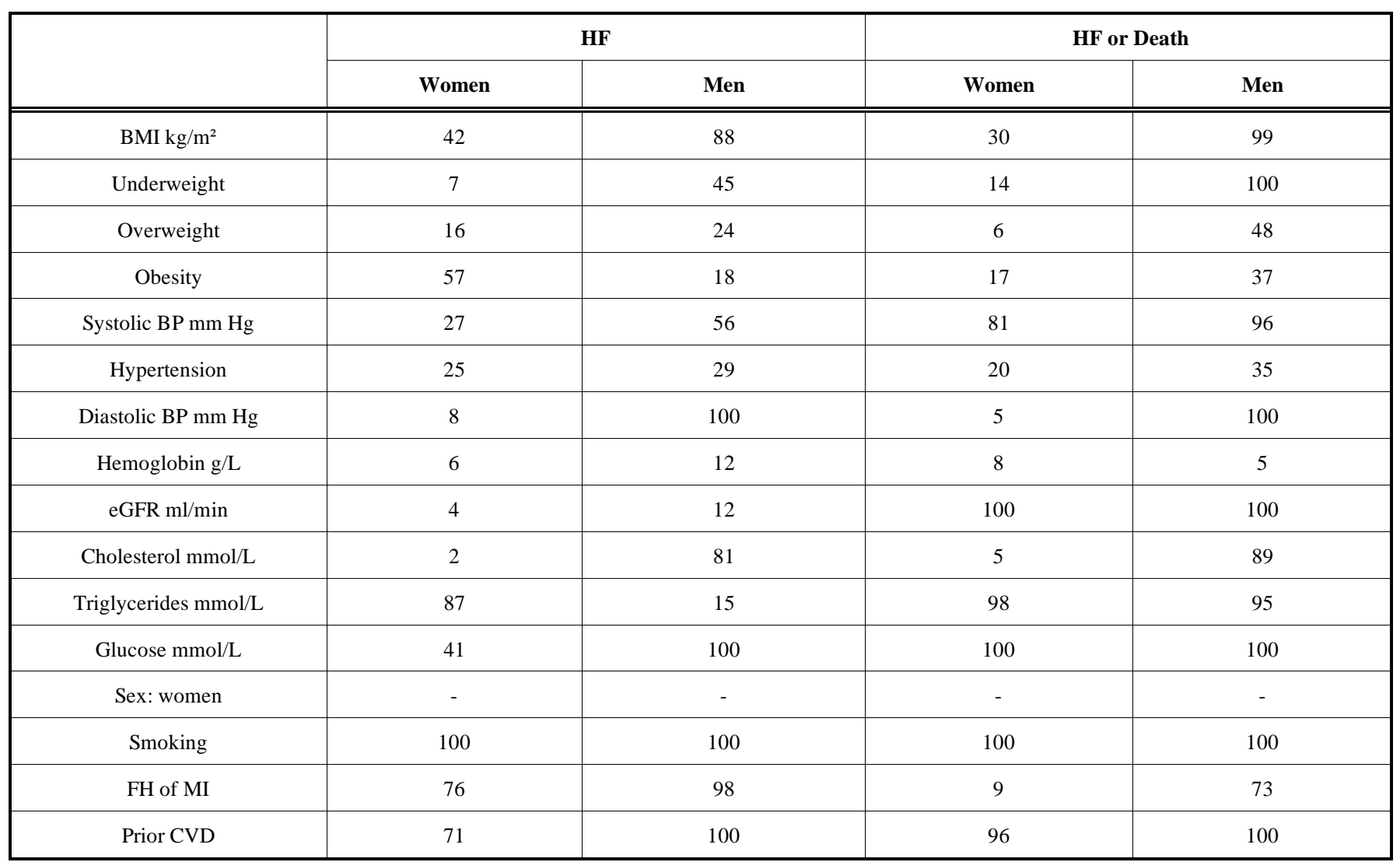

HF, Heart failure; BMI, Body mass index; BP, Blood pressure; Smoking, current and previous smokers versus never smokers; FH of MI, Family history of acute myocardial infarction; CVD, Cardiovascular disease, eGFR (Estimated glomerular filtration rate). 
Table 4a. Multivariable Analysis of Strong Independent Predictors of Heart Failure and Combined Heart Failure or Death, According to Bootstrap Analysis, in Women and Men (Adjusted for Age)

\begin{tabular}{|c|c|c|c|c|c|c|c|c|c|c|c|c|}
\hline & \multicolumn{6}{|c|}{ Predictors of $\mathbf{H F}$} & \multicolumn{6}{|c|}{ Predictors of HF or Death } \\
\hline & HR & $95 \% \mathrm{CI}$ & $\mathbf{P}$ & HR & $95 \% \mathrm{CI}$ & $\mathbf{P}$ & HR & $95 \% \mathrm{CI}$ & $\mathbf{P}$ & HR & $95 \% \mathrm{CI}$ & $\mathbf{P}$ \\
\hline $\mathrm{BMI} \mathrm{kg} / \mathrm{m}^{2}$ & - & - & - & - & - & - & - & - & - & 1.04 & $1.03-1.05$ & $<.0001$ \\
\hline Underweight & - & - & - & - & - & - & - & - & - & 1.36 & $1.23-1.50$ & $<.0001$ \\
\hline Overweight & - & - & - & - & - & - & - & - & - & - & - & - \\
\hline Systolic BP mm Hg & - & - & - & - & - & - & - & - & - & 1.01 & $1.00-1.01$ & $<.0001$ \\
\hline Hypertension & - & - & - & - & - & - & - & - & - & - & - & - \\
\hline Diastolic BP mm Hg & - & - & - & 1.04 & $1.04-1.05$ & $<.0001$ & - & - & - & 1.01 & $1.01-1.02$ & $<.0001$ \\
\hline Hemoglobin $\mathrm{g} / \mathrm{L}$ & - & - & - & - & - & - & - & - & - & - & - & - \\
\hline eGFR ml/min & - & - & - & - & - & - & 1.01 & $1.01-1.02$ & $<.0001$ & 1.001 & $1.001-1.003$ & $<.0001$ \\
\hline Sex: Women & - & - & - & - & - & - & - & - & - & - & - & - \\
\hline Smoking & 2.52 & $1.72-3.69$ & $<.0001$ & 1.92 & $1.62-2.27$ & $<.0001$ & 2.05 & $1.80-2.34$ & $<.0001$ & 2.16 & $2.03-2.31$ & $<.0001$ \\
\hline FH of MI & - & - & - & 1.40 & $1.15-1.70$ & $<.0001$ & - & - & - & - & - & - \\
\hline Prior CVD & - & - & - & 4.14 & $2.44-7.00$ & $<.0001$ & 2.21 & $1.41-3.45$ & $<.0001$ & 2.28 & $1.81-2.88$ & $<.0001$ \\
\hline
\end{tabular}

HF, Heart failure; BMI, Body mass index; BP, Blood pressure; Smoking, current and previous smokers versus never smokers; FH of MI, Family history of acute myocardial infarction; CVD, Cardiovascular disease, eGFR (Estimated glomerular filtration rate).HRs for continuous variables are per unit increase, except for BMI, BP and eGFR, where HRs are per 10 units increase. HRs for the baseline BMI categories are for the respective categories versus the normal weight category. HRs for all other categories are compared to the opposite category.

Table 4b. Multivariable Analysis of Weak and Strong Independent Predictors of Heart Failure and Combined Heart Failure or Death, According to Bootstrap Analysis, in Women and Men (Adjusted for Age)

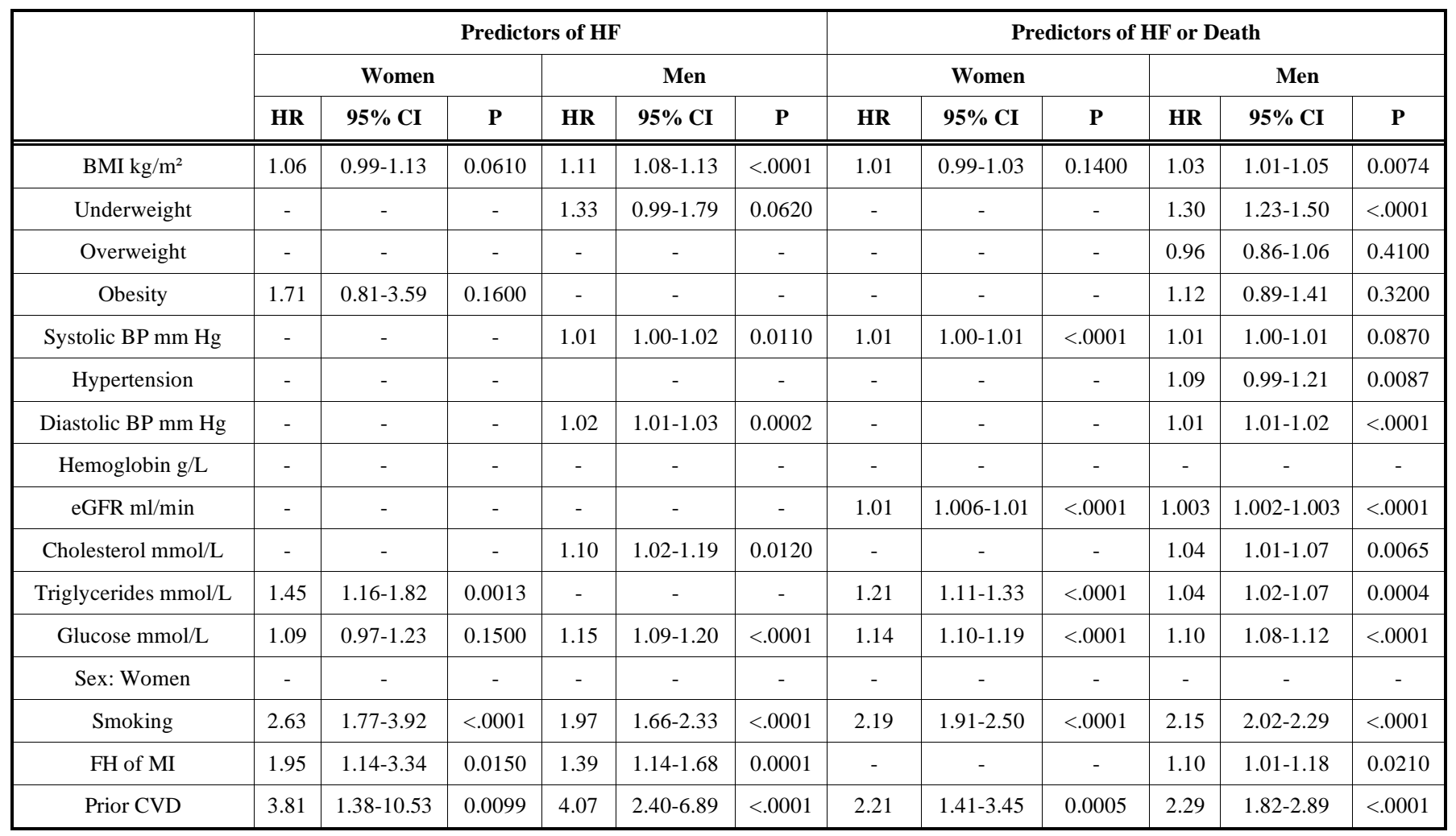

HF, Heart failure; BMI, Body mass index; BP, Blood pressure; Smoking, current and previous smokers versus never smokers; FH of MI, Family history of acute myocardial infarction; CVD, Cardiovascular disease, eGFR (Estimated glomerular filtration rate).

HRs for continuous variables are per unit increase, except for BMI, BP and eGFR, where HRs are per 10 units increase. HRs for the baseline BMI categories are for the respective categories versus the normal weight category. HRs for all other categories are compared to the opposite category. 
Since few patients died of HF (5 women and 27 men), we did not assess predictors of HF death.

\section{Predictors of HF or Death}

Among women, bootstrap analysis showed that strong independent predictors of HF or death were high eGFR, striglycerides and fb-glucose, as well as smoking, and prior CVD, whereas high BMI and systolic BP were weak independent predictors (Table 3). In the final multivariable model including strong independent predictors, high eGFR, s-triglycerides and fb-glucose, as well as smoking, and prior CVD were independent. In addition to these variables, high systolic BP was independent in the final multivariable model including both strong and weak independent predictors of HF or death among women (Table $\mathbf{4 b}$ ).

In bootstrap analysis among men, underweight, high BMI, systolic BP, diastolic BP, eGFR, s-triglycerides and fbglucose, as well as smoking, and prior CVD, were strong independent predictors of HF or death (Table 3). Overweight, obesity, hypertension, high s-cholesterol, and family history of MI, were weak independent predictors (Table $\mathbf{3}$ ). In the final multivariable models, all strong independent predictors were independent, except that systolic BP was not independent in the final model including both strong and weak independent predictors (Tables $4 \mathbf{a}$ and $\mathbf{b}$ ). All weak independent predictors were independent in the final multivariable model including both strong and weak independent predictors, except overweight and obesity (Table 4b).

\section{DISCUSSION}

In the present study, we assessed predictors of HF and, for the sake of competing risks, the combined endpoint of HF or all-cause death. The predictors were assessed at baseline, among 33,342 middle-aged men and women of a Swedish community with approximately 275,000 inhabitants. At baseline, subjects were on average around 46 years of age, and since they were followed-up for a mean of approximately 22 years, subjects were on average still middle-aged at study end.

Few studies have examined predictors of HF. In the American study, NHANES I [16], male sex, low education, physical inactivity, smoking, overweight, hypertension, diabetes, valvular disease and ischemic heart disease were independent predictors of developing HF, whereas high scholesterol was not. Obesity has been reported to be among the strongest predictors of HF. In recently published analyses from Framingham [20], moderate overweight (BMI 25-30 $\mathrm{kg} / \mathrm{m}^{2}$ ) was an independent predictor of developing HF among women but not among men. However, those who had BMI over $30 \mathrm{~kg} / \mathrm{m}^{2}$ had twice the risk of developing HF among both sexes, compared to those with normal BMI. A similar risk increase associated with obesity was seen in a Swedish study of middle-aged men [21].

The predictors of HF in the present study compare reasonably well with those of prior studies, although with some noteworthy differences $[16,20]$. Thus, we found that, besides age, strong independent predictors of developing HF among men were high diastolic BP and fb-glucose, as well as smoking, family history of MI and previous CVD. However, among women, the only strong independent predictor of HF was smoking. Interestingly, and in contrast to the
Framingham study [20], neither overweight, nor obesity were independent predictors of HF in either sex. However, obesity was a weak independent predictor among women and high BMI (as a continuous variable) was a weak independent predictor of HF among both sexes, and almost qualified as a strong independent predictor among men. Further, in contrast to men, high diastolic BP and s-cholesterol were not independent predictors of HF among women. Previous studies have shown that diabetes mellitus is an important predictor of CVD and HF among both men and women, although more important among women [29]. In contrast, in the present study, high fb-glucose was a strong independent predictor of HF only among men, whereas it was only a weak independent predictor among women, not even independent in the final multivariable model. This could potentially be explained by the relatively young mean age of subjects in our study.

Underweight, high BMI, systolic BP, diastolic BP, eGFR, s-triglycerides and fb-glucose, as well as smoking and previous CVD, were strong independent predictors of combined HF or death among men. In contrast, underweight, high BMI, and systolic and diastolic BP, were not strong independent predictors among women. Many studies have shown a relationship between decreased eGFR and increased risk of CVD and CVD death [30]. In the present study, eGFR was not at all an independent predictor of HF alone, whereas increasing eGFR was a strong independent predictor of combined HF or death among both women and men. This rather surprising finding may be due to other reasons than actual renal function. S-creatinine poorly reflects renal function in individuals with normal to slightly decreased renal function. Using the MDRD formula, a low s-creatinine results in a high eGFR. However, a low s-creatinine may be due to reduced muscle mass, which may be the true reason for the poor prognosis, e.g. if it is caused by chronic disease. We believe that a low s-creatinine was predominantly a sign of reduced muscle mass in our study. Furthermore, the method of calculating eGFR by the MDRD formula was developed from a study of 1628 patients with chronic renal disease [24]. The appropriateness of this equation to estimate renal function in large groups of patients to study the effect of renal function on cardiovascular risk factors has, however, been debated. Because the MDRD equation was developed from a study that did not include healthy subjects it has raised concern of underestimating GFR in healthier populations. In a study by Rule et al. of 320 patients with chronic kidney disease and 580 healthy kidney donors, it was reported that the MDRD equation underestimated GFR by $6.2 \%$ in patients with chronic kidney disease and by 29 $\mathrm{ml} / \mathrm{min} / 1.73 \mathrm{~m}^{2}$ in the healthy group [31]. Since our patients had normal or close to normal renal function according to the s-creatinine values, it is wise not to draw any firm conclusions regarding the relationship between eGFR and the outcome endpoint of HF or death.

We have not done any formal p-value adjustment for multiple statistical testing [32]. However, anyone is free to make their own judgment as to whether $\mathrm{p}<0.05$ or e.g. $<0.01$ should denote statistical significance, and consider independent predictors accordingly. With rather few exceptions, most independent predictors showed $p<0.01$ in multivariable analysis. 


\section{STUDY LIMITATIONS}

Importantly, the present study included subjects who were relatively young at baseline and who were followed-up for the limited time period of 22 years, at which time point they were on average still quite young. This is illustrated by the observation that only around $16 \%$ of all subjects died during follow-up, $9 \%$ of the women and $20 \%$ of the men. The predictors may change during life and may be different at a later stage in life. Further, predictors may be different among subjects of a mean age of around 65 years at the time of endpoint assessment, as in the present study, as compared with subjects of a more advanced age. Therefore, our results may not be universally applicable. The HF diagnosis, as well as a lack of a HF diagnosis, may not be entirely correct. However, our internal validation suggests that it is largely correct. Patients who were diagnosed with HF without any hospitalisation or death during follow-up were not identified as HF patients. However, it is unlikely that a patient with HF will survive for many years without any hospitalisation. We have not assessed social, ethnic or educational background of the subjects, which may be regarded as a limitation. We did not have general access to data on cardiac function, e.g. LVEF, which also is a limitation. Lastly, we do not know if there was any selection bias of our subjects at the time of inclusion.

In conclusion, while independent predictors of developing HF were similar to the classical predictors of CVD among men, women differed quite substantially in this regard. This was also largely true for independent predictors of combined HF or all-cause death.

\section{ACKNOWLEDGEMENTS}

The Swedish Heart-Lung Foundation supported this study. We thank all subjects participating in the MPP and the staff at the research department who carried out the examinations.

\section{REFERENCES}

[1] Bush TL. The epidemiology of cardiovascular disease in postmenopausal women. Ann NY Acad Sci 1990; 592: 263-71.

[2] Stampfer MJ, Colditz GA, Willett WC. Menopause and heart disease. A review. Ann NY Acad Sci 1990; 592: 193-203.

[3] Barret-Connor E. Sex differences in coronary heart disease. Why are women so superior? The 1995 Ancel Keys Lecture. Circulation 1997; 95: 252-64.

[4] Yach D, Hawkes C, Gould CL, et al. The global burden of chronic disease: overcoming impediments to prevention and control. JAMA 2004; 291: 2616-22.

[5] Leal J, Luengo-Fernadez R, Gray A, et al. Economic burden of cardiovascular diseases in the enlarged European Union. Eur Heart J 2006; 27: 1610-9.

[6] De Backer G, Ambrosioni E, Borch-Johnsen K, et al. European guidelines on cardiovascular disease prevention in clinical practice. Third joint task force of European and other societies on cardiovascular disease prevention in clinical practice. Eur Heart J 2003; 24: 1601-10.

[7] Wenger NK. Hypertension and other cardiovascular risk factors in women. Am J Hypertens 1995; 8: 94-9.

[8] Neaton JD, Blackburn H, Jacobs D, et al. Serum cholesterol level and mortality findings for men screened in the multiple risk factor intervention trial. Multiple risk factor intervention trial research group. Arch Intern Med 1992; 152: 1490-1500.
Thomas F, Bean K, Guize L, et al. Combined effects of systolic blood pressure and serum cholesterol on cardiovascular mortality in young men end women. Eur Heart J 2002; 23: 528-35.

[10] Wilhelmsen L, Lappas G, Rosengren A. Risk of coronary events by baseline factors during 28 years follow-up and three periods in a random population sample of men. J Intern Med 2004; 256: 298307.

[11] Andresdottir MB, Sigurdsson G, Sigvaldason H, et al. Reykjavik cohort study. Fifteen percent of myocardial infarctions and coronary revascularizations explained by family history unrelated to conventional risk factors. The Reykjavik Cohort Study. Eur Heart J 2002; 23: 1655-63.

[12] Yusuf S, Hawken S, Ounpuu S, et al. INTERHEART study investigators. Effect of potentially modifiable risk factors associated with myocardial infarction in 52 countries (the INTERHEART study): case-control study. Lancet 2004; 364: 937-52.

[13] Rosengren A, Hawken S, Ounpuu S, et al. Association of psychosocial risk factors with risk of acute myocardial infarction in 11119 cases and 13648 controls from 52 countries (the INTERHEART study): case-control study. Lancet 2004; 364: 953-62.

[14] Schnohr P, Jensen JS, Scharling H, et al. Coronary heart disease risk factors ranked by importance for the individual and community. A 21 year follow-up of 12000 men and women from the copenhagen city heart study. Eur Heart J 2002; 23: 620-6.

[15] Nilsson PM, Nilsson JA, Berglund G. Population-attributable risk of coronary heart disease risk factors during long-term follow-up: the Malmö Preventive Project. J Intern Med 2006; 260: 134-41.

[16] He J, Ogden LG, Bazzano LA, et al. Risk factors for congestive heart failure in US men and women: NHANES I epidemiologic follow-up study. Arch Intern Med 2001; 161: 996-1002.

[17] Lee DS, Massaro JM, Wang TJ, et al. Antecedent blood pressure, body mass index, and the risk of incident heart failure in later life. Hypertension 2007; 50: 869-76.

[18] Fonarow GC, Srikanthan P, Costanzo MR, et al. ADHERE Scientific Advisory Committee and Investigators. An obesity paradox in acute heart failure: analysis of body mass index and in hospital mortality for 108,927 patients in the Acute Decompensated Heart Failure National Registry. Am Heart J 2007; 153: 74-81.

[19] Thrainsdottir IS, Aspelund T, Gudnason V, et al. Increasing glucose levels and BMI predict future heart failure experience from the Reykjavík Study. Eur J Heart Fail 2007; 9: 1051-7.

[20] Kenchaiah S, Evans JC, Levy D, et al. Obesity and risk of heart failure. N Engl J Med 2002; 347: 305-13.

[21] Wilhelmsen L, Rosengren A, Eriksson H, et al. Heart failure in the general population of men-morbidity, risk factors and prognosis. J Intern Med 2001; 249: 253-61.

[22] Berglund G, Nilsson P, Eriksson K-F, et al. Long-term outcome of the Malmö Preventive Project: mortality and cardiovascular morbidity. J Intern Med 2000; 247: 19-29.

[23] Kennedy LM, Dickstein K, Anker SD, et al. Weight-change as a prognostic marker in 12550 patients following acute myocardial infarction or with stable coronary artery disease. Eur Heart J 2006; 27: 2755-62.

[24] Levey AS, Bosh JP, Lewis JB, et al. A more accurate method to estimate glomerular filtration rate from serum creatinine: a new prediction equation. Modification of diet in renal disease study Group 1999; 130: 461-70.

[25] Efron B, Tibshirani R. Bootstrap methods for standard errors, confidence intervals and other measures of statistical accuracy. Stat Sci 1986; 1: 54-77.

[26] Burr D. A comparison of certain bootstrap confidence intervals in the Cox regression model. J Am Stat Assoc 1994; 29: 1290-1302.

[27] Sauerbrei W. The use of resampling methods to simplify regression models in medical statistics. J R Stat Soc Ser C 1999; 48: 313-29.

[28] Grambsch P, Therneau T. Proportional hazards tests and diagnostics based on weighted residuals. Biometrika 1994; 515-26.

[29] Kannel WB. Incidence and epidemiology of heart failure. Heart Fail Rev 2000; 5: 167-73. 
[30] Sarnak M, Levey A. Cardiovascular disease and chronic renal disease: A new paradigm. Am J Kidney Dis 2000; 35: 117-31.

[31] Rule AD, Gussak HM, Pond GR, et al. Measured and estimated GFR in healthy potential kidney donors. Am J Kidney Dis 2004; 43: 112-119.
[32] Perneger T. Whats wrong with Bonferroni adjustments. BMI 1998; 316: $1236-38$

Received: September 25, 2008

(C) Tasevska-Dinevska et al.; Licensee Bentham Open.

This is an open access article licensed under the terms of the Creative Commons Attribution Non-Commercial License (http://creativecommons.org/licenses/by-nc/3.0/) which permits unrestricted, non-commercial use, distribution and reproduction in any medium, provided the work is properly cited. 